\title{
Observations of the Lyman series forest towards the redshift 7.1 quasar ULAS J1120+0641
}

\author{
R. Barnett ${ }^{1}$, S. J. Warren ${ }^{1}$, G. D. Becker ${ }^{2}$, D. J. Mortlock ${ }^{1,3,4}$, P. C. Hewett ${ }^{5}$, R. G. McMahon ${ }^{5,6}$, \\ C. Simpson ${ }^{7}$, and B. P. Venemans ${ }^{8}$
}

\author{
1 Astrophysics Group, Blackett Laboratory, Imperial College London, London SW7 2AZ, UK \\ e-mail: rhys. barnett09@imperial.ac.uk \\ 2 Department of Physics \& Astronomy, University of California, Riverside, 900 University Avenue, Riverside, CA 92521, USA \\ 3 Department of Mathematics, Imperial College London, London SW7 2AZ, UK \\ 4 Department of Astronomy, Stockholm University, Albanova, 10691 Stockholm, Sweden \\ 5 Institute of Astronomy, University of Cambridge, Madingley Road, Cambridge CB3 OHA, UK \\ ${ }^{6}$ Kavli Institute for Cosmology, University of Cambridge, Madingley Road, Cambridge CB3 OHA, UK \\ 7 Gemini Observatory, Northern Operations Center, N. A'ohoku Place, Hilo, HI 96720, USA \\ 8 Max-Planck Institut für Astronomie, Königstuhl 17, 69117 Heidelberg, Germany
}

Received 15 December 2016 / Accepted 9 February 2017

\begin{abstract}
We present a $30 \mathrm{~h}$ integration Very Large Telescope X-shooter spectrum of the Lyman series forest towards the $z=7.084$ quasar ULAS J1120+0641. The only detected transmission at $S / N>5$ is confined to seven narrow spikes in the Ly $\alpha$ forest, over the redshift range $5.858<z<6.122$, just longward of the wavelength of the onset of the Ly $\beta$ forest. There is also a possible detection of one further unresolved spike in the $\operatorname{Ly} \beta$ forest at $z=6.854$, with $S / N=4.5$. We also present revised Hubble Space Telescope $F 814 \mathrm{~W}$ photometry of the source. The summed flux from the transmission spikes is in agreement with the $F 814 W$ photometry, so all the transmission in the Lyman series forest may have been detected. There is a Gunn-Peterson (GP) trough in the Ly $\alpha$ forest from $z=6.122$ all the way to the quasar near zone at $z=7.04$. The trough, of comoving length $240 \mathrm{~h}^{-1} \mathrm{Mpc}$, is over twice as long as the next longest known GP trough. We combine the spectroscopic and photometric results to constrain the evolution of the Ly $\alpha$ effective optical depth $\left(\tau_{\mathrm{GP}}^{\mathrm{eff}}\right)$ with redshift, extending a similar analysis by Simpson et al. We find $\tau_{\mathrm{GP}}^{\mathrm{eff}} \propto(1+z)^{\xi}$ where $\xi=11.2_{-0.6}^{+0.4}$, for $z>5.5$. The data nevertheless provide only a weak limit on the volume-weighted intergalactic medium (IGM) hydrogen neutral fraction at $z \sim 6.5, x_{\mathrm{HI}}>10^{-4}$, similar to limits at redshift $z \sim 6$ from less distant quasars. The new observations cannot extend measurements of the neutral fraction of the IGM to higher values because absorption in the Ly $\alpha$ forest is already saturated near $z \sim 6$. For higher neutral fractions, other methods such as measuring the red damping wing of the IGM will be required.
\end{abstract}

Key words. cosmology: observations - quasars: individual: ULAS J1120+0641 - quasars: absorption lines dark ages, reionization, first stars

\section{Introduction}

It is now over 50 years since Gunn \& Peterson (1965, GP) noted the lack of Lyman- $\alpha$ (Ly $\alpha)$ absorption in the spectra of (at the time) high redshift quasars. The cross-sections of the Lyman series transitions are so high that even a small neutral hydrogen fraction $\left(x_{\mathrm{HI}} \sim 10^{-4}\right)$ should result in an intergalactic medium (IGM) that is effectively opaque to photons at wavelengths shorter than that of Ly $\alpha$ emission, $1215.67 \AA$ A However, using observations of the $z=2.01$ quasar 3C 9 (Schmidt 1965), Gunn \& Peterson (1965) were able to demonstrate an extremely low density of neutral hydrogen $\left(x_{\mathrm{H}}<10^{-5}\right)$ in the IGM near $z=2$.

More recently, the $\operatorname{Ly} \alpha$ forest has been used to constrain the end of reionization by attempting to measure the extent of $\operatorname{Ly} \alpha$ absorption at redshifts of around six. The lack of GP troughs in the spectra of SDSS quasars with $z<6$ (e.g. Fan et al. 2000, 2001; Djorgovski et al. 2001; Songaila \& Cowie 2002) indicated that the IGM was highly ionized, since a low volume weighted neutral fraction $\left(\sim 10^{-4}\right)$ would be sufficient to result in complete absorption of Ly $\alpha$ photons. The first GP trough was reported by Becker et al. (2001) towards the $z=6.28$ quasar
SDSS J1010+0524 (see also White et al. 2003). They concluded that the neutral fraction must increase sharply at $z \sim 6$.

A seminal study of Lyman series absorption was that of Fan et al. (2006a, see also Fan et al. 2006b), who presented the largest individual sample of high redshift quasars to date. From the spectra of $19 z \sim 6$ sources, they noted a sharp increase in the effective optical depth to Ly $\alpha$ photons $\left(\tau_{\mathrm{GP}}^{\text {eff }}\right)$. Their conclusion was that there is an acceleration of the evolution of the IGM ionization state at $z \gtrsim 5$.7. Importantly, Fan et al. (2006a) reported a large line-of-sight dependence, indicating patchiness in the IGM neutral fraction that could be related to cosmic reionization. Fan et al. (2006a) also measured a dramatic increase in the average length of dark gaps (in this case defined as contiguous regions with optical depth $\tau>3.5$ ) for $z>6$, suggesting that Ly $\alpha$ troughs could be a powerful probe of the IGM at higher redshifts.

The detection of a $\sim 110 h^{-1}$ Mpc GP trough covering the redshift range $5.523 \leq z \leq 5.879$ in the $\mathrm{Ly} \alpha$ forest towards the $z=5.98$ quasar ULAS J0148+0600 by Becker et al. (2015) recently strengthened the case for patchiness. Comparing the observations to hydrodynamical simulations, they argue that significant fluctuations in the hydrogen neutral fraction as late as $z \simeq$ 5.6-5.8 are required to explain the data. 


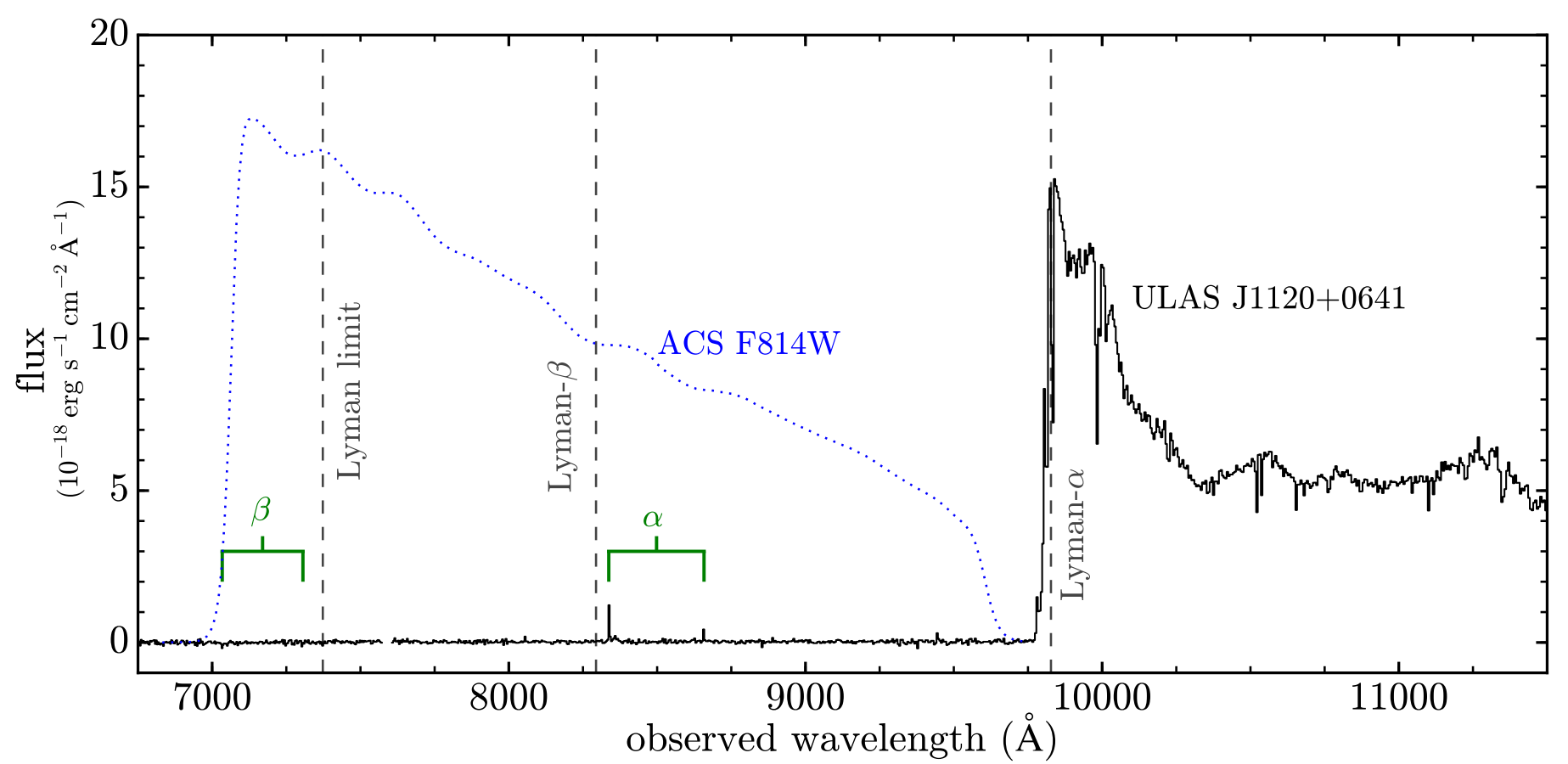

Fig. 1. VLT X-shooter spectrum of ULAS J1120+0641 (black), covering restframe wavelengths between 850 and $1400 \AA$ A. The spectrum has been binned to a pixel size of $150 \mathrm{~km} \mathrm{~s}^{-1}$ using inverse variance weighting. The observed wavelengths of Ly $\alpha$, Ly $\beta$ and the Lyman limit are marked. The only transmission detected in the spectrum is confined to spikes over the wavelength range marked by $\alpha$, in the pure Ly $\alpha$ forest, corresponding to the redshift range $5.858<z<6.122$ (Sect. 3.2). The corresponding wavelength range for Ly $\beta$ absorption is marked $\beta$ and lies blueward of the restframe Lyman limit. Plotted as the blue dotted line is the (scaled) total system throughput curve for the ACS F814W filter.

Measurements of dark pixels in the $\operatorname{Ly} \alpha$ and $\operatorname{Ly} \beta$ forests have also been used to place the first model independent constraints on reionization. Rather than attempting to measure optical depths based on a continuum model, Mesinger (2010) proposed a novel approach, where the fraction of pixels that have zero measured flux in both $\operatorname{Ly} \alpha$ and $\operatorname{Ly} \beta$ is used to directly measure an upper limit to the volume-weighted mean neutral hydrogen fraction. Applying the idea to observed spectra, McGreer et al. $(2011,2014)$ conclude that reionization must be (almost) complete by $z \sim 6$.

The most recent optical depth measurements from Planck, integrated to the last scattering surface $(z \sim 1100)$, yield $\tau=$ $0.055 \pm 0.009$, suggesting a model-dependent redshift of reionization between $\sim 7.8$ and $\sim 8.8$ (Planck Collaboration XLVI; Planck Collaboration XLVII 2016).

These various results both from spectroscopic analyses and Planck motivate extending observations of $\operatorname{Ly} \alpha$ absorption to higher redshifts. ULAS J1120+0641 (Mortlock et al. 2011) remains the only known quasar with $z>7(z=7.084$; Venemans et al. 2012). In this paper we analyse the Ly $\alpha$ forest towards ULAS J1120+0641 by combining Hubble Space Telescope (HST) imaging from the Advanced Camera for Surveys (ACS), first presented by Simpson et al. (2014), with new spectroscopy from the Very Large Telescope (VLT)/X-shooter instrument (Vernet et al. 2011). The spectrum of ULAS J1120+0641 is shown in Fig. 1. In Sect. 2 we present the data, paying close attention to the effects of charge transfer efficiency (CTE) losses in the case of HST. We remeasure the ACS F814W (i-band) image of the quasar. In Sect. 3 we analyse the X-shooter spectrum. We discuss the limitations of spectroscopy for determining optical depth measurements towards the source, and search for significant transmission in the spectrum. In Sect. 4 we combine our photometric and spectroscopic measurements in order to constrain the evolution of effective optical depth towards the quasar, and use simulations to set limits on $x_{\mathrm{HI}}$. We summarise in Sect. 5.

All magnitudes are on the AB system (Oke \& Gunn 1983). We have adopted a concordance cosmology with $h=0.7, \Omega_{\mathrm{M}}=$ 0.3 , and $\Omega_{\Lambda}=0.7$.

\section{Observations and data reduction}

\subsection{VLT X-shooter spectroscopy}

The source was observed with the $\mathrm{X}$-shooter spectrograph over several nights spanning 2011 March to 2014 April, providing a total of $30 \mathrm{~h}$ integration on source ${ }^{1}$. The Visual-red (VIS) arm of X-shooter, with MIT/LL CCD detectors, spans the wavelength range 5500-10000 $\AA$, and the Near-IR arm (NIR), with a Hawaii $2 R G$ array detector, spans the wavelength range $10000-$ $25000 \AA$, or $1.0-2.5 \mu \mathrm{m}$. The quasar's Ly $\alpha$ emission line lies at $9827 \AA$, close to the boundary between the two arms. In the current paper we analyse the Lyman series absorption in the spectrum, i.e., the wavelength range from the quasar Lyman limit at $7371 \AA$ up to the Ly $\alpha$ emission line. This wavelength range lies entirely within the VIS arm.

The data reduction, including flux calibration, is described more fully in Bosman et al. (2017), who provide the spectrum redward of $\operatorname{Ly} \alpha$, and present the results of a search for metal lines in that region. Briefly, sky subtraction was performed using a custom IDL implementation of the optimum sky subtraction routines outlined in Kelson (2003). This approach involves transforming the $(x, y)$ CCD coordinates into a wavelength and

1 ESO programmes 286.A-5025(A), 089.A-0814(A), and 093.A0707(A). 
slit position for each pixel in the two-dimensional frame. Slit positions for each order were defined using a standard star trace. In orders where the quasar has little flux, such as in the Ly $\alpha$ forest, the offset from the standard trace and the spatial profile of the object were extrapolated from orders where flux is present, assuming that there is minimal atmospheric dispersion between orders $^{2}$. This assumption is reasonable given that the Ly $\alpha$ forest for ULAS J1120+0641 falls at fairly red wavelengths, and because the object was generally observed at moderate airmass (less than 1.5). Optimal techniques (Horne 1986) were used to extract a one-dimensional spectrum simultaneously from all exposures for a given spectrograph arm after a relative flux calibration was applied to each order in the two-dimensional frames.

Great care was taken to minimise bias in the sky-subtraction stage of the data reduction, since we are interested in measuring the degree of absorption in the Lyman series forest relative to the continuum. Systematics, where the sky level to subtract is consistently over- or under-estimated, are more important for ULAS J1120+0641 than for several of the other high-redshift quasars used for measuring transmission in the Ly $\alpha$ forest because the source is fainter. For example, three quasars in which long GP troughs have been detected, ULAS J0148+0600, $z=$ 5.98 (Becker et al. 2015), and SDSS J1030+0524, $z=6.31$ and SDSS J1148+5251, $z=6.42$ (White et al. 2003), are respectively factors 3.9, 2.1, and 2.9 brighter than ULAS J1120+0641. Subtle systematics can be present depending on the algorithm used. For example, a weighted least squares fit to the sky where the variance is a Poisson estimate based on the measured counts will systematically underestimate the sky and so leave a positive residual (see White et al. 2003, for a related discussion). To avoid such a bias, we instead used a least-squares linear fit. Likewise, combining multiple exposures using inverse variance weighting can produce a small positive bias when the variance is calculated using a Poisson estimate based on amplitude of the sky fit (plus a contribution from read noise). The bias occurs because exposures in which the sky is underestimated (and hence the object flux overestimated) at a particular wavelength will be assigned a lower variance, and hence a larger weight. To mitigate this bias, we estimated the variance as a function of wavelength using the measured scatter in the two-dimensional counts about the sky fit. A Poisson contribution from the object, based on the extracted counts, is also added along the object trace.

The observations were made with a 0.9 slit width, providing a nominal resolving power of $R=8800$, or a resolution of $34 \mathrm{~km} \mathrm{~s}^{-1}$. Inspection of the telluric absorption lines, however, indicates that the true mean resolving power was somewhat better, $R \sim 10000$, or a resolution of $30 \mathrm{~km} \mathrm{~s}^{-1}$, as a consequence of good average seeing during the observations. The bin size used for the final spectrum is $10 \mathrm{~km} \mathrm{~s}^{-1}$. The spectrum was flux calibrated using observations of a spectrophotometric standard. Due to slit losses the zero point (i.e. overall scaling) will be uncertain. Accordingly the spectrum was then scaled by matching to the GNIRS spectrum presented in Mortlock et al. (2011) over the region of overlap, redward of the Ly $\alpha$ forest, 9800$10000 \AA$. The GNIRS spectrum itself was calibrated to UKIRT photometry, from 2011. The quasar was observed in 2013 March with HST (Sect. 2.2) in the F814W, F105W, and F125W filters (Simpson et al. 2014), and found to have faded by $0.16 \mathrm{mag}$. compared to the UKIRT $Y J$ photometry. Because we use the F814W photometry in our analysis of the Ly $\alpha$ forest, we have applied this additional flux correction to the spectrum, meaning

\footnotetext{
2 The majority of our data were acquired in 2013 and 2014, when the $\mathrm{X}$-shooter atmospheric dispersion corrector was not in use.
}

that the normalization differs from that used by Bosman et al. (2017).

The new X-shooter spectrum is presented in Fig. 1, binned to a pixel size of $150 \mathrm{~km} \mathrm{~s}^{-1}$. As detailed below, the only detectable transmission over the entire Lyman series is confined to the small range in wavelengths marked by $\alpha$ in Fig. 1, corresponding to the redshift range 5.858-6.122, in the pure Ly $\alpha$ forest, and just redward of the onset of the $\operatorname{Ly} \beta$ forest, at $\lambda_{\text {obs }}=8291.92 \AA$, $z=5.821$. The remainder of the $\operatorname{Ly} \alpha$ forest from $z=6.122$ up to the edge of the quasar near zone at $z=7.04$ is completely absorbed, as is the entire region of the $\operatorname{Ly} \beta, \gamma$ and higher series, down to the Lyman limit. The bin width of $150 \mathrm{~km} \mathrm{~s}^{-1}$ in Fig. 1 was chosen to emphasise the GP absorption in the plot.

We checked the computed error spectrum by analysing the distribution of signal-to-noise ratio $(\mathrm{S} / \mathrm{N})$ in pixels in the long GP trough. We first subtracted off any large-scale systematic residual (Sect. 3.1) using a 301 pixel median filter, before calculating the S/Ns. We applied a $3 \sigma$ clip and fit a Gaussian to the resulting histogram, which should have a dispersion of unity if the errors are correct. We bin the data for this particular analysis, e.g., by factors of 2, 4, etc., to account for the slight smoothing at the pixel-to-pixel level introduced by rebinning at the wavelength calibration stage. Scaling the noise array by a factor of 1.05 resulted in a Gaussian fit to the residuals with a dispersion of unity. As noted by Bosman et al. (2017), the sky subtraction is imperfect close to the peak of the brightest $\mathrm{OH}$ sky lines. Again fitting a Gaussian to the $\mathrm{S} / \mathrm{N}$ histogram for pixels close to the peak of strong lines, we were able to treat the imperfect sky subtraction by further increasing the errors by a factor of 1.15 for the five pixels centred on each skyline peak.

The errors are on average substantially smaller than in the spectra of the three quasars listed earlier. For example compared to ULAS J1120+0641, in the GP troughs in the spectra of ULAS J0148+0600, $z=5.98$, SDSS J1030+0524, $z=6.31$, and SDSS J1148+5251, $z=6.42$, measured over the same wavelength regions, the errors are on average larger by factors 1.6 , 2.5 , and 1.8 , respectively (consistent with the difference in integration times).

\subsection{HST imaging}

HST observed the field of ULAS J1120+0641 with the ACS and WFC 3 instruments in the three filters $F 814 \mathrm{~W}, F 105 \mathrm{~W}$, and F125W, during 2013 March (Simpson et al. 2014). As shown in Fig. 1 the wavelength range of the ACS $F 814 W$ filter rather neatly spans the Lyman series forest of ULAS J1120+0641, and so photometry of ULAS J1120+0641 in this filter provides valuable information on the Lyman series absorption. The $F 814 \mathrm{~W}$ image had a total exposure time of $28448 \mathrm{~s}$ spread across five visits made over 2013 March 27-29; it is substantially deeper than the Subaru $i^{\prime}$-band image presented in Barnett et al. (2015). Each visit comprised six dithered exposures, with the exception of the final visit where a single exposure was taken. The 25 pipeline-processed frames were then combined using the PYRAF routine astrodrizzle.

Simpson et al. (2014) measured $i_{814}=28.59 \pm 0.20$ for ULAS J1120+0641 with aperture photometry, using the result to constrain the evolution of the effective optical depth towards the quasar (Sect. 4.1). The source is close to the limit of detectability, and in ACS images the effect of CTE losses can be important for such faint sources. As the counts in a detected source are clocked down to the serial register, some charge gets left behind and appears in a trail after the source (Anderson \& Bedin 2010; 
Table 1. Measured flux density in GP troughs.

\begin{tabular}{ccccc}
\hline \hline Quasar & Redshift range & $\begin{array}{c}\text { Wavelength range } \\
\AA\end{array}$ & $\begin{array}{c}\text { Flux density } \\
10^{-20} \mathrm{erg} \mathrm{s}^{-1} \mathrm{~cm}^{-2} \AA^{-1}\end{array}$ & $\begin{array}{c}\text { ULAS J1120+0641 } \\
10^{-20} \mathrm{erg} \mathrm{s}^{-1} \mathrm{~cm}^{-2} \AA^{-1}\end{array}$ \\
\hline ULAS J0148+0600 & $5.55-5.71$ & $7966-8163$ & $-0.3 \pm 0.9$ & $2.2 \pm 0.5$ \\
ULAS J0148+0600 & $5.71-5.88$ & $8163-8360$ & $0.9 \pm 0.8$ & $2.7 \pm 0.5$ \\
SDSS J1030+0524 & $6.00-6.16$ & $8510-8710$ & $1.5 \pm 1.4$ & $3.2 \pm 0.5$ \\
SDSS J1148+5251 & $6.10-6.32$ & $8630-8900$ & $3.5 \pm 0.8$ & $2.7 \pm 0.5$ \\
\hline
\end{tabular}

Notes. The final column represents the flux measured over the same wavelength range for ULAS J1120+0641, with significant transmission spikes subtracted.

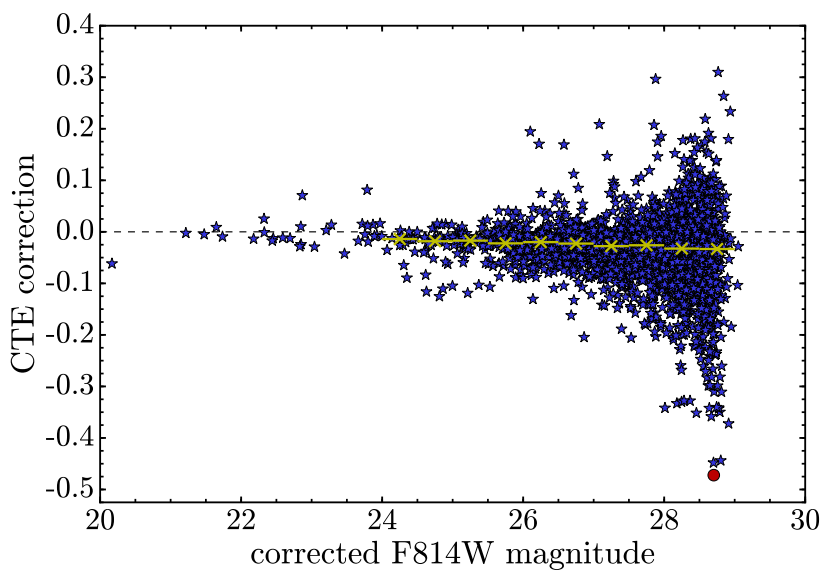

(a)

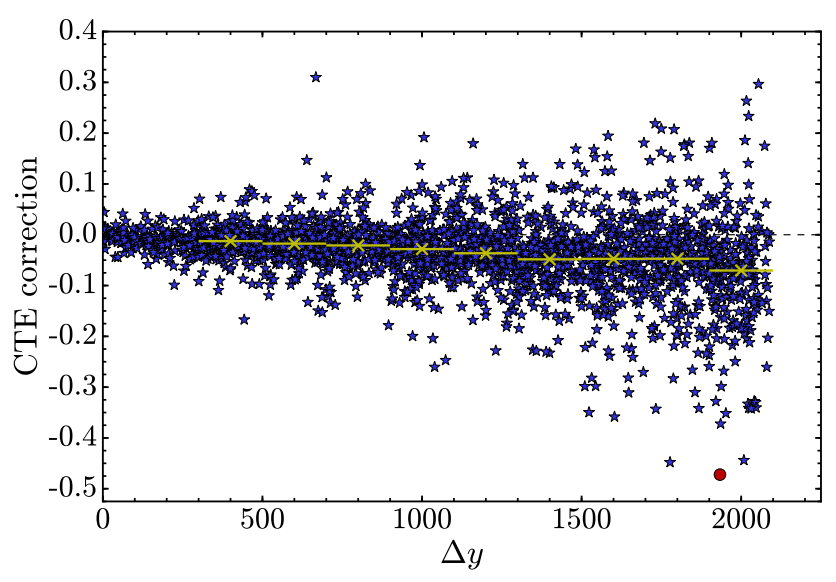

(b)

Fig. 2. CTE correction $\left(m_{\text {corrected }}-m_{\text {uncorrected }}\right)$ applied to objects detected in the F814W frame, shown as blue stars. The red circle indicates ULAS J1120+0641. Yellow crosses represent the median correction in the indicated bins of $0.5 \mathrm{mag}$ (upper) or 200 pixels (lower). Upper: correction as a function of corrected $F 814 \mathrm{~W}$ magnitude. Lower: correction as a function of $\Delta y$, the number of pixels from the readout register.

Chiaberge 2012). The problem is more severe the lower the background and proportionally greatest for the faintest sources, increasing linearly with distance of the source from the serial register, i.e., with the parallel clocking distance. The placement of ULAS J1120+0641 close to the centre of the field of view of the ACS instrument unfortunately means that the object lies at the edge of one of the CCDs. The distance to the serial register is maximised, as is the charge loss. Therefore, it is important to assess the effect of CTE losses on the photometry.
Two solutions to the problem of CTE losses have been devised by the ACS team. The first (Chiaberge 2012) quantifies the losses as a function of sky background, source brightness, and location on the CCD, and provides formulae to correct measured photometry. The second (Anderson \& Bedin 2010) attempts to put the trailed charge back into the sources, by applying an ingenious algorithm that uses a model for the CCD traps (defects) that cause the CTE losses. The latter method has proved so successful that it is now applied by default in the ACS data reduction pipeline (although there is an option to turn it off), and the image measured by Simpson et al. (2014) was corrected in this manner.

ULAS J1120+0641 is located close to the ecliptic, resulting in a comparatively bright sky background. In a single frame the average sky level in our F814W frames is $\sim 105 \mathrm{e}^{-}$. This is a considerably higher background than the levels explored by Chiaberge (2012). As the background level rises the CTE corrections become smaller, and the coefficients provided by Chiaberge (2012) allow one to estimate the background level at which the CTE correction becomes zero. Although it becomes necessary to extrapolate their results, the level is $\sim 70 \mathrm{e}^{-}$, implying that any CTE corrections in our frames should actually be very small. We consulted with the ACS instrument team on the issue of the CTE correction, and they recommended that at such high background levels losses should indeed be small, and that we should use frames without the Anderson \& Bedin (2010) corrections applied, since for high background the algorithm will simply add noise.

As a check, we used astrodrizzle to produce co-added frames of the dithered F814W exposures both with and without the Anderson \& Bedin (2010) correction in order to quantify the CTE corrections made as a function of source brightness, and of location on the CCD. We paid close attention to the choice of input parameters to astrodrizzle to avoid the cores of bright objects being masked as cosmic rays. We used SEXTRACTOR (Bertin \& Arnouts 1996) to produce a catalogue of objects in the HST frames, detected in the corrected F814W image. We performed aperture photometry at the positions specified by the catalogue in both the corrected and uncorrected F814W images. Ideally the sample would be restricted to point sources, but star/galaxy discrimination is unreliable for sources as faint as ULAS J1120+0641, so we included all catalogued sources. CTE corrections for galaxies will be larger than for point sources, as they cover more pixels, so any measured trends may in fact overestimate the CTE correction for a point source. The centroid of the image of ULAS J1120+0641 is also uncertain as the source is so faint. We therefore used the F105W frame to define the position of the quasar, before measuring photometry in the two F814W frames. However, the F105W proved a poor choice to define the whole catalogue, as it is shallower than the F814W observation and so fewer objects were detected. Finally, we calculated the CTE corrections $\left(\Delta m=m_{\text {corrected }}-m_{\text {uncorrected }}\right)$ 
for all sources. These are presented as a function of corrected magnitude in Fig. 2a, and of the distance in pixels from the serial register, $\Delta y$, in Fig. 2b. In each of these plots very weak trends, at the level of few hundredths of a magnitude are visible in the mean correction for all objects at a particular magnitude or clocking distance, but these are much smaller than the standard deviation. Furthermore, as noted above, these trends may overestimate the required correction, as the sample is dominated by galaxies. We conclude that when the background is this high the Anderson \& Bedin (2010) algorithm simply introduces additional noise, and the best photometry is provided by the uncorrected frame. The correction for ULAS J1120+0641 itself is large: the quasar lies in the tail of the distributions and is brightened by $0.47 \mathrm{mag}$. This unusually large correction appears to be due to chance.

The uncorrected frame has a stripy appearance from (fractionally small) CTE losses in bright sources. Therefore, we flattened the sky background before aperture photometry by subtracting a median-filtered version of the frame. We measured the counts in a 3-pixel radius (rather than the 2.5 pixel aperture preferred by Simpson et al. 2014, to minimise digitisation issues), and applied an aperture correction to 10 pixel radius $\left(00^{\prime \prime} 5\right)$, derived from bright stars. This was then further corrected to the nominal "infinite" aperture radius of the ACS of 5".5 using the quoted encircled energy fraction of 0.914 in the 0 ".5 aperture, quoted in the ACS Data Handbook. The final photometry result is $i_{814}=28.85$, with $S / N=2.9$. We quote the error as a signalto-noise ratio as the errors are Gaussian in flux, but at such low significance the equivalent posterior distribution in magnitudes is extremely skewed, and we do not quote it here.

\section{Analysis of the spectrum}

\subsection{Systematic errors in sky subtraction}

In exploring the Ly $\alpha$ forest in the new spectrum, smoothing on different scales, we found that despite the care taken in the data reduction to minimise such effects (Sect. 2.1), there are systematic errors present in the form of a small positive residual signal at an average level of $f_{\lambda} \sim 3 \times 10^{-20} \mathrm{erg} \mathrm{s}^{-1} \mathrm{~cm}^{-2} \AA^{-1}$. The excess corresponds to only $\sim 0.05 \%$ of the counts from the sky within the extraction aperture, or $\sim 0.15$ of the average random error per pixel. We used SYNPHOT (Laidler et al. 2008) to measure synthetic photometry from the spectrum, obtaining a value $i_{814}=27.2$, which is a factor 4.5 brighter than the HST photometry. The low-level sky residual is difficult to characterise quantitatively, since a $3 \sigma$ detection alone requires binning over 400 pixels $(\sim 120 \AA)$. However, the residual does not correlate directly with the error spectrum, in the sense that on large scales they do not share the same shape. Neither is the residual significantly greater in the wavelength ranges of the bands of $\mathrm{OH}$ sky lines, compared to the line-free regions. Therefore it does not seem to be an effect associated with the difficulty of subtracting the sharp OH sky lines, and is apparently a smooth feature.

Systematic errors in sky subtraction have not been reported or quantified in previous measurements of the Lyman-series effective optical depth. We checked whether a residual at the level seen in the spectrum of ULAS J1120+0641 would have been detected in the GP troughs seen in the spectra of the three quasars ULAS J0148+0600 (Becker et al. 2015), SDSS J1030+0524, and SDSS J1148+5251 (White et al. 2003). In these papers, the transmission in the GP troughs is measured in bins of width $\sim 50 \mathrm{Mpc}$, and transformed to an effective optical depth. Using the same bins we have measured the average flux density in the spectra, using inverse-variance weighting, and the results are provided in the fourth column of Table 1 . In three of the bins the measured flux is consistent with zero, while significant flux is recorded in the GP trough $6.10<z<6.32$ in the spectrum of SDSS J1148+5251. We then measured the average flux density over the same four bins in the spectrum of ULAS J1120+0641, after subtracting the few transmission spikes seen in the spectrum (Sect. 3.2), in order to measure the smooth residual. These results are listed in the final column of Table 1 . In the spectrum of ULAS J1120+0641 we measure significant flux at the level of $\sim 3 \times 10^{-20} \mathrm{erg} \mathrm{s}^{-1} \mathrm{~cm}^{-2} \AA^{-1}$ in all four bins.

The detection of significant flux in the redshift bin $6.10<$ $z<6.32$ in the spectrum of SDSS J1148+5251 is interesting, because it is the only other case where significant flux has been measured where no transmission spikes in Ly $\alpha$ have been detected. In the original paper, White et al. (2003) speculated that this was due to flux from a faint intervening galaxy, a suggestion which has subsequently been ruled out by HST imaging (White et al. 2005). Systematic errors in the sky subtraction may provide a simpler explanation. In fact, comparing the values in Cols. 4 and 5 of Table 1, in each bin the measurements agree at the $2.5 \sigma$ level. This means that systematic errors could be present in the other spectra at a similar level, and the reason they are reported here for the first time is because it is the deepest spectrum yet taken at these redshifts.

\subsection{Detection of transmission spikes}

To remove the systematic residual we subtracted a medianfiltered version of the spectrum using a box of length 301 pixels, i.e., $\sim 90 \AA$. At these redshifts transmission in the Lymanseries forest will be in isolated spikes, so the application of a median filter of such a long box length should have a negligible effect on any inferred transmission. The region of the spectrum covering the Ly $\alpha$ forest, binned by a factor two for display purposes, i.e., pixels of $20 \mathrm{~km} \mathrm{~s}^{-1}$, is shown in Fig. 3, together with the error spectrum. Note, however, that we use the unbinned spectrum to search for regions of significant transmission. To search for spikes in the Lyman series forest $\left(7370 \AA \leq \lambda_{\text {obs }} \leq\right.$ $9770 \AA$ ) we employ a Gaussian matched filter (Hewett et al. 1985; Bolton et al. 2004), with a range of widths. The narrowest profile has a standard deviation $\Sigma=15 \mathrm{~km} \mathrm{~s}^{-1}$, corresponding to the nominal spectral resolution of $34 \mathrm{~km} \mathrm{~s}^{-1} F W H M$. A range of profiles successively increasing in width by $\sqrt{2}$ was employed, $\Sigma=15,21,30, \ldots, 120 \mathrm{~km} \mathrm{~s}^{-1}$. We fit a Gaussian at every pixel, 8535 in total. To illustrate the process we plot the $S / N$ at each pixel for the narrowest template in the bottom panel of Fig. 3. Then, for each pixel, we recorded the $S / N$ of the most significant detection over all the templates, whether positive or negative, and the corresponding value of $\Sigma$.

The distribution of these $S / N$ values is shown in Fig. 4. This distribution is non-Gaussian, because we recorded the maximum absolute value for each pixel. Since negative spikes, which are not real, extend down to nearly $S / N=-4$, we cannot be sure positive spikes are real unless the $S / N$ is substantially greater than 4 . In fact one might expect a somewhat more extended tail in the positive direction, of spikes that are not real, because of cosmic rays that are not perfectly subtracted. For these reasons we set our detection threshold at $S / N=5$. The matched filter finds seven significant flux transmission spikes, at observed wavelengths $8337 \AA \leq \lambda_{\text {obs }} \leq 8659 \AA$, redshifts $5.858<z<6.122$. These are summarised in Table 2 and plotted in Fig. 5. These seven spikes are also identifiable in both panels 


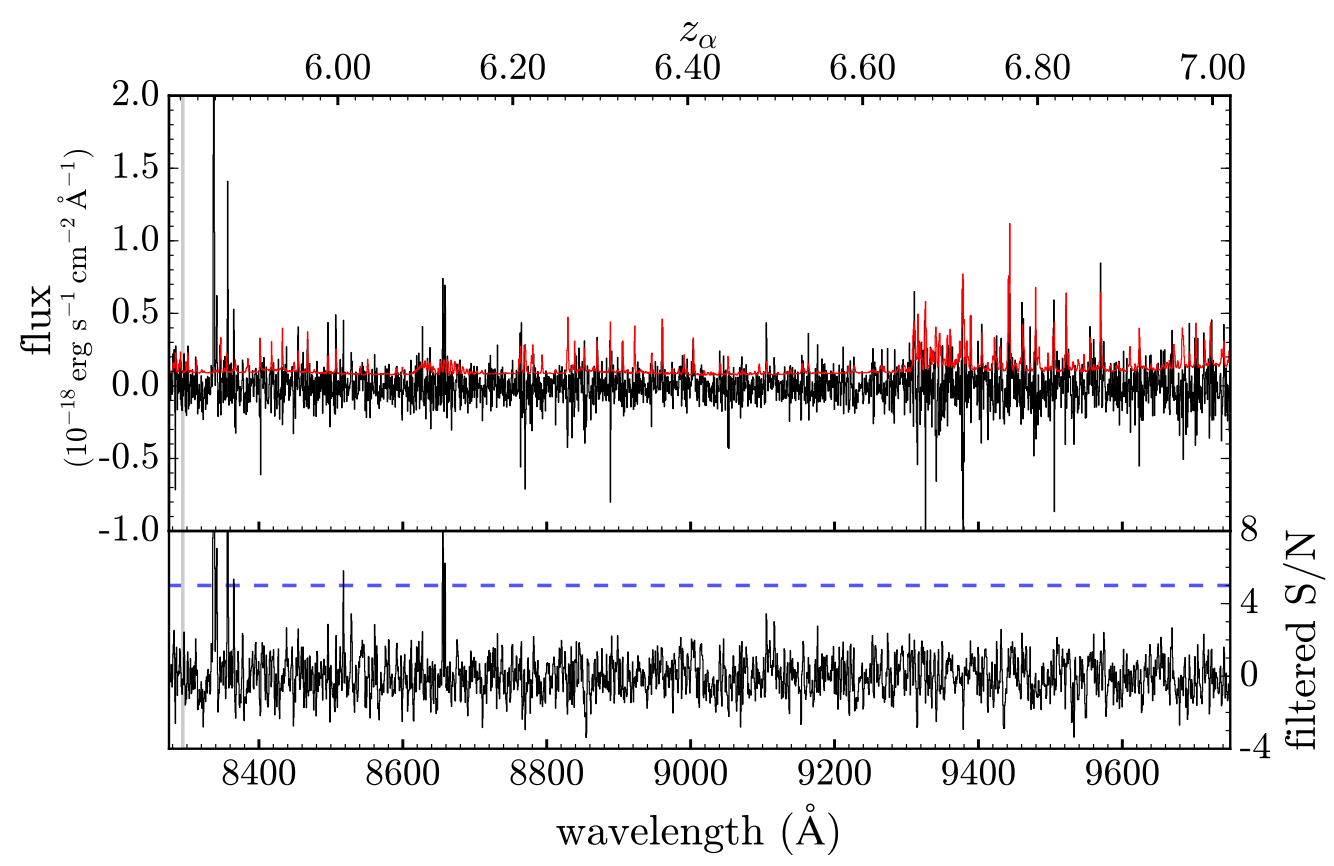

Fig. 3. Close-up of the Ly $\alpha$ forest towards ULAS J1120+0641, binned to a pixel size of $20 \mathrm{~km} \mathrm{~s}^{-1}$ for display purposes. Upper: spectrum (black) and $1 \sigma$ errors (red). Lower: $S / N$ at each pixel found by running the Gaussian matched filter at the narrowest width over the unbinned spectrum (pixel size $10 \mathrm{~km} \mathrm{~s}^{-1}$ ).

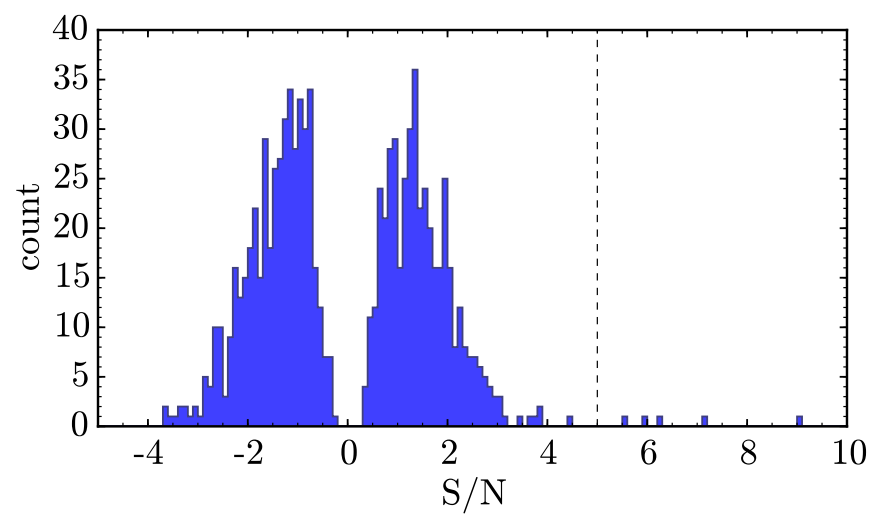

Fig. 4. $S / N$ of spikes extracted from the X-shooter spectrum. We have extracted the highest value of $|\mathrm{S} / \mathrm{N}|$ from all template widths.

of Fig. 3. To confirm that the detections are real, for each spike we compared the measured flux at the detected wavelength in subsets of the data, and found no significant differences between subsets. We also checked the goodness of the fit of the Gaussian template to the data, finding the $\chi^{2}$ values to be satisfactory. The observed transmission features are very narrow: with the exception of the largest spike at $8337 \AA$, the template width $\Sigma$ which yielded the highest $S / N$ was $15 \mathrm{~km} \mathrm{~s}^{-1}$, i.e., the spikes are unresolved. We integrate the template transmission spectrum to find a total transmitted flux of $(1.02 \pm 0.03) \times 10^{-17} \mathrm{erg} \mathrm{s}^{-1} \mathrm{~cm}^{-2}$, of which approximately half is contained in the largest spike.

Using SYNPHOT we measure $i_{814}=29.00 \pm 0.03$ for these seven spikes. Our value is consistent with the HST photometry (Sect. 2.2; $i_{814}=28.85, S / N=2.9$ ), raising the question of whether the seven spikes account for all the transmission in the spectrum. In considering this question we refer to Table 3 which sets out the wavelength ranges in the spectrum and the corresponding redshifts over which absorption in the four transitions
Table 2. Transmission spikes detected in the $\operatorname{Ly} \alpha$ forest with the matched filter.

\begin{tabular}{ccccc}
\hline \hline $\begin{array}{c}\lambda_{\text {obs }} \\
\AA\end{array}$ & $z_{\alpha}$ & $\begin{array}{c}\Sigma \\
\mathrm{km} \mathrm{s}^{-1}\end{array}$ & $S / N$ & $\begin{array}{c}\text { Spike flux } \\
10^{-18} \mathrm{erg} \mathrm{s}^{-1} \mathrm{~cm}^{-2}\end{array}$ \\
\hline 8337.2 & 5.858 & 21 & 48.7 & 5.34 \\
8341.4 & 5.862 & 15 & 7.4 & 0.71 \\
8356.7 & 5.874 & 15 & 14.8 & 1.42 \\
8365.1 & 5.881 & 15 & 5.6 & 0.54 \\
8517.4 & 6.006 & 15 & 5.9 & 0.46 \\
8655.7 & 6.120 & 15 & 9.0 & 0.84 \\
8658.6 & 6.122 & 15 & 6.2 & 0.85 \\
\hline
\end{tabular}

Ly $\alpha, \beta, \gamma, \delta$ are recorded. It is noticeable that the seven spikes all lie close to the blue end of the pure Ly $\alpha$ forest, which is to be expected. Starting at the red end of the Ly $\alpha$ forest, on the edge of the quasar near zone (near $9770 \AA$ ), and moving to shorter wavelengths, the Ly $\alpha$ opacity reduces, down to $8291 \AA$, $z_{\alpha}=5.82$, where the high-opacity $\operatorname{Ly} \beta$ forest at $z_{\beta}=7.08$ cuts in. Moving to shorter wavelengths from $8291 \AA$ the opacity in $\operatorname{Ly} \beta$ becomes lower, but will still be very high at $7862 \AA$, corresponding to $z_{\beta}=6.61$, at which point the Ly $\gamma$ forest cuts in at $z_{\gamma}=7.08$. The result is that the most transparent window in the spectrum is just redward of $8291 \AA$. Therefore if there is any more transmitted flux in the spectrum, which will be in the form of narrow spikes below $5 \sigma$ significance, most of it is expected to lie in the wavelength range 8291-8700 ̊. Looking at the spikes with $S / N$ in the range 3-5 these are approximately uniformly distributed along the spectrum, with no excess detected in the wavelength range $8291-8700 \AA$. Even if all the $3-5 \sigma$ spikes in this wavelength range were real, they would only contribute an additional $10 \%$ to the transmitted flux. The region of $\operatorname{Ly} \beta$ absorption corresponding to the transmission spikes, is marked $\beta$ in Fig. 1 . It is 

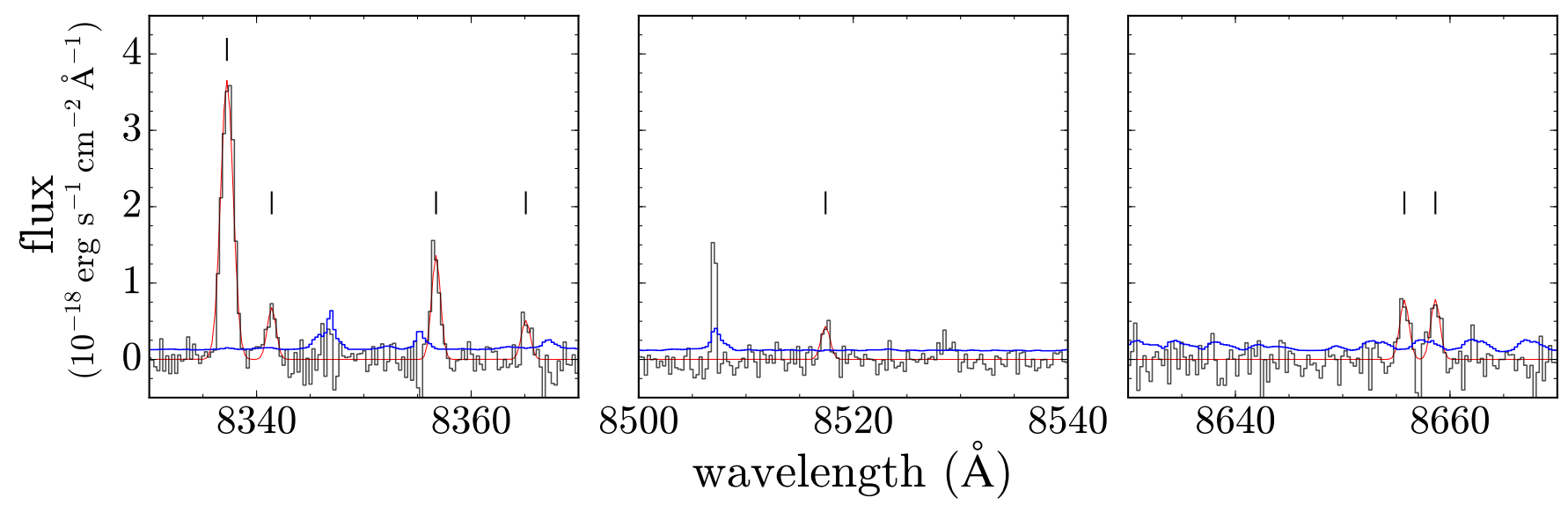

Fig. 5. Significant transmission of flux in the Ly $\alpha$ forest towards ULAS J1120+0641. The unbinned X-shooter spectrum of ULAS J1120+0641 is shown in grey with a pixel size of $10 \mathrm{~km} \mathrm{~s}^{-1}$. Shown in blue is the $1 \sigma$ error spectrum, and the derived transmission spectrum is overlaid in red. The seven detections presented in Table 2 are indicated.

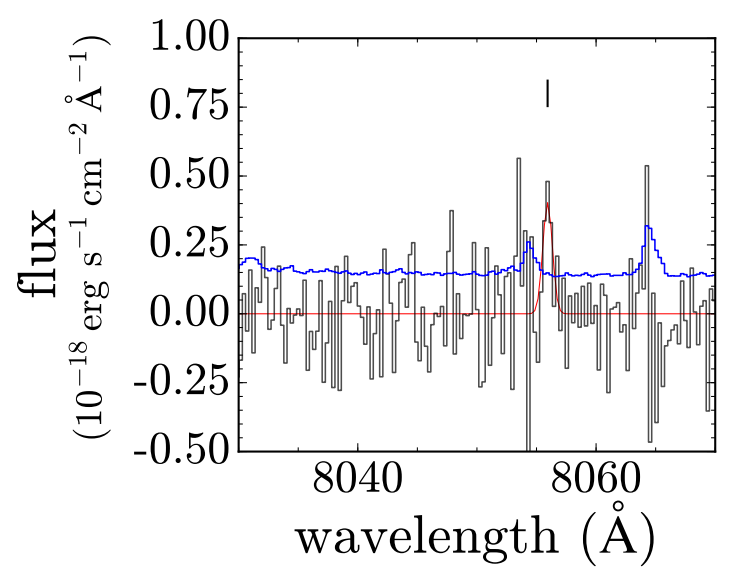

Fig. 6. As Fig. 5, but focused on the region of the spectrum in the $\operatorname{Ly} \beta$ forest in the vicinity of the possible transmission spike at $\lambda=8056 \AA$, which is indicated.

Table 3. Wavelength and redshift ranges recording $\operatorname{Ly} \alpha, \beta, \gamma, \delta$ absorption.

\begin{tabular}{ccccc}
\hline \hline $\begin{array}{c}\lambda_{\text {obs }} \\
\AA\end{array}$ & $\alpha$ & $\beta$ & $\gamma$ & $\delta$ \\
A & & & & \\
\hline $8291-9827$ & $5.82-7.08$ & - & - & - \\
$7862-8291$ & $5.47-5.82$ & $6.61-7.08$ & - & - \\
$7678-7862$ & $5.32-5.47$ & $6.49-6.66$ & $6.89-7.08$ & - \\
$7581-7678$ & $5.24-5.32$ & $6.39-6.49$ & $6.80-6.89$ & $6.98-7.08$ \\
\hline
\end{tabular}

beyond the quasar Lyman limit, and is consequently not useful for measuring transmission.

There is a single unresolved spike in the range of ambiguity $4<S / N<5$. It is detected at $S / N=4.5$, and lies at $\lambda=$ $8056 \AA$, corresponding to $z_{\alpha}=5.627$ and $z_{\beta}=6.854$. The spike is plotted in Fig. 6. A region of transmission at $z=6.854$ would be very unexpected given the rapid evolution of the hydrogen neutral fraction seen around $z \sim 6$, so the feature is potentially interesting. We checked that the fluxes measured in subsets of the data are consistent. The putative line lies six pixels from the centre of a strong OH sky line, sufficiently far away that it should not be affected by poor sky subtraction. Unfortunately it is not possible to be sure if the line is real or not.
If the spike is real, it requires that the opacities along the line of sight to the quasar at redshifts $z_{\alpha}=5.627$ and $z_{\beta}=6.854$ are both low. We are able to quantify this statement by following a line of reasoning used by White et al. (2005) in discussing a $\operatorname{Ly} \beta$ transmission spike in the spectrum of SDSS J1148+5251. In the spectrum of ULAS J1120+0641, the fractional transmission measured over five pixels centred on the $\operatorname{Ly} \beta$ spike is $\mathcal{T}_{8056}=0.04 \pm 0.01$. The effective optical depth at $8056 \AA$, which is the sum of contributions from both the $\operatorname{Ly} \alpha$ and $\operatorname{Ly} \beta$ forests, is $\tau_{8056}=3.2 \pm 0.3=\tau_{\alpha}(z=5.6)+\tau_{\beta}(z=6.9)$. The transmission at the corresponding wavelength in the Ly $\alpha$ forest, $z_{\alpha}=6.854$, $\lambda=9548 \AA$, measured over 5 pixels, is $\mathcal{T}(9548)=-0.01 \pm 0.01$, meaning an optical depth $\tau_{\alpha}(z=6.9)>4.0$ at $2 \sigma$. Taking the ratio of effective optical depths $\tau_{\alpha} / \tau_{\beta} \sim 2.25$ from Fan et al. (2006a), the measurement at $\lambda=9548 \AA$, implies $1.8<\tau_{\beta}(z=$ $6.9)<3.2$, and $0<\tau_{\alpha}(z=5.6)<1.4$. The range on $\tau_{\alpha}(z=5.6)$ is not unreasonable, meaning that our analysis does not weaken the case that the line is real. Combining the two transmission measurements, and assuming $\tau_{\alpha} / \tau_{\beta} \sim 2.25$, leads to the constraint $4.0<\tau_{\alpha}(z=6.9)<7.2$ for this spike.

\subsection{Measurement of the effective GP optical depth}

Narrow features are essentially unaffected by the application of the long median filter, and so we use the filtered spectrum to measure the effective optical depth in the Lyman series forest. To normalise the spectrum and hence estimate the transmitted flux ratio $\mathcal{T}$, we divide the $\mathrm{X}$-shooter spectrum by the model intrinsic spectrum described in Mortlock et al. (2011). Here the blue wing of the Ly $\alpha$ emission line comes from a composite spectrum created from sources with similar CIV blueshifts, and the spectrum is extended bluewards as a power law $F_{\lambda} \propto \lambda^{-0.5}$.

In Table 4 we present measurements of $\mathcal{T}$ and effective GP optical depth $\tau_{\mathrm{GP}}^{\text {eff }}$ over the redshift range 5.07-7.02, covering practically the entire Lyman series forest. We follow the prescription of Fan et al. (2006a), making measurements in fixedsize bins of $\Delta z=0.15$. The redshift bin 6.87-7.02 was excluded as the median filtered spectrum is affected by the quasar Ly $\alpha$ emission line in this region. To estimate $\mathcal{T}$ we calculate the transmission fraction in each pixel before taking the inverse-variance weighted average for each bin, i.e., $\mathcal{T}=\left\langle F / F_{\text {cont }}\right\rangle$. If we detect 
Table 4. Measurements of the transmission fraction $\mathcal{T}$ and effective optical depth $\tau_{\mathrm{GP}}^{\mathrm{eff}}$ in bins of $\Delta z=0.15$ towards ULAS J1120+0641.

\begin{tabular}{cccccc}
\hline \hline Wavelength range & Redshift range & \multicolumn{2}{c}{ Bin size } & $\mathcal{T}$ & $\tau_{\mathrm{GP}}^{\text {eff }}$ \\
$\AA$ & $z_{\alpha}$ & pixels & $h^{-1} c \mathrm{Mpc}$ & & \\
\hline $7381.3-7563.5$ & $5.07-5.22$ & 732 & 53.6 & $0.00000 \pm 0.00088$ & $>6.34$ \\
$7563.7-7745.8$ & $5.22-5.37$ & 635 & 51.7 & $-0.00114 \pm 0.00110$ & $>6.12$ \\
$7746.0-7928.2$ & $5.37-5.52$ & 698 & 49.9 & $0.00111 \pm 0.00093$ & $>6.29$ \\
$7928.5-8110.7$ & $5.52-5.67$ & 682 & 48.3 & $0.00067 \pm 0.00093$ & $>6.29$ \\
$8110.9-8292.9$ & $5.67-5.82$ & 666 & 46.6 & $0.00015 \pm 0.00082$ & $>6.41$ \\
$8293.1-8475.5$ & $5.82-5.97$ & 653 & 45.2 & $0.00640 \pm 0.00095$ & $5.05 \pm 0.15$ \\
$8475.8-8657.8$ & $5.97-6.12$ & 638 & 43.7 & $0.00013 \pm 0.00084$ & $>6.38$ \\
$8658.1-8840.2$ & $6.12-6.27$ & 625 & 42.4 & $-0.00023 \pm 0.00091$ & $>6.31$ \\
$8840.5-9022.5$ & $6.27-6.42$ & 612 & 41.1 & $0.00055 \pm 0.00094$ & $>6.28$ \\
$9022.8-9204.9$ & $6.42-6.57$ & 600 & 39.9 & $-0.00058 \pm 0.00086$ & $>6.37$ \\
$9205.2-9387.5$ & $6.57-6.72$ & 589 & 38.8 & $0.00071 \pm 0.00118$ & $>6.05$ \\
$9387.8-9569.6$ & $6.72-6.87$ & 576 & 37.5 & $-0.00019 \pm 0.00114$ & $>6.09$ \\
\hline
\end{tabular}

Notes. Lower limits on $\tau_{\mathrm{GP}}^{\mathrm{eff}}$ correspond to twice the uncertainty on $\mathcal{T}$, if the measurement of $\mathcal{T}$ is below $2 \sigma$.

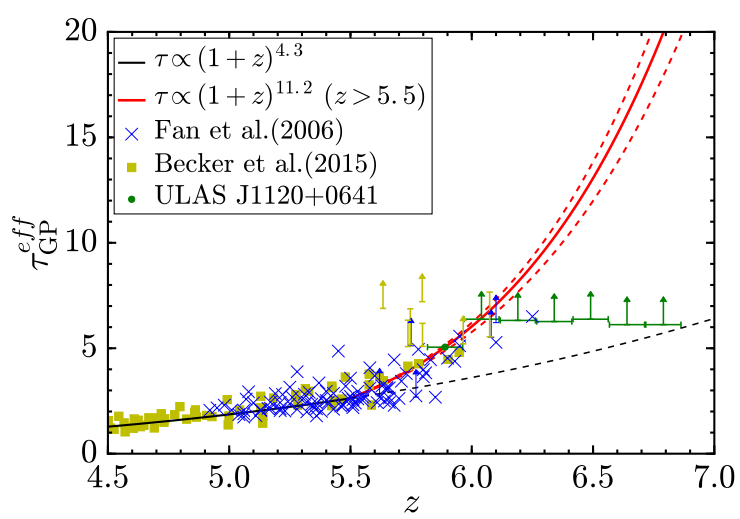

Fig. 7. Evolution of $\tau_{\mathrm{GP}}^{\mathrm{eff}}$ with redshift as predicted by Eq. (1) (solid lines). The red curve is determined using ULAS J1120+0641 data alone and assumes a fixed normalization at $z=5.5$. The uncertainty in $\xi$ for $z>5.5$ is indicated by the red dashed lines. The black dashed curve indicates the expected optical depth if the low-redshift case were to continue indefinitely. Points indicate direct measurements of $\tau_{\mathrm{GP}}^{\text {eff }}$ along different lines of sight.

transmission below a level of $2 \sigma$, we adopt a lower limit for $\tau_{\mathrm{GP}}^{\mathrm{eff}}$ based on a value of $\mathcal{T}$ equal to twice the uncertainty ${ }^{3}$.

We also consider the effect of the adopted power-law slope at wavelengths below Ly $\alpha$. We recalculate $\tau_{\mathrm{GP}}^{\text {eff }}$ values using a bluer continuum, $F_{\lambda} \propto \lambda^{-1.4}$ (Lusso et al. 2015). The effect on $\tau_{\mathrm{GP}}^{\text {eff }}$ is found to be small. In the lowest-redshift bin we find $\tau_{\mathrm{GP}}^{\mathrm{eff}}>6.54$, while in the highest redshift bins the difference is negligible. In the bin beginning at $z=5.82$ we measure $\tau_{\mathrm{GP}}^{\mathrm{eff}}=5.15 \pm 0.15$, i.e., consistent with the value presented in Table 4.

In Fig. 7 we show our measurements of $\tau_{\mathrm{GP}}^{\mathrm{eff}}$, as well as the power law evolution of $\tau_{\mathrm{GP}}^{\mathrm{eff}}$ determined using HST photometry (Sect. 4.1). We do not include the bins for which $z_{\alpha}<5.82$ in Fig. 7, since these have contributions from $\operatorname{Ly} \beta$ and higher order

\footnotetext{
3 A more elegant approach would be to deduce a posterior probability density function for $\mathcal{T}$ and hence $\tau_{\mathrm{GP}}^{\text {eff }}$. The true value of $\mathcal{T} \geq 0$, so by assuming a uniform prior for $\mathcal{T} \geq 0$ and a Gaussian likelihood, it would be possible to determine posterior confidence intervals for $\tau_{\mathrm{GP}}^{\text {eff }}$. By adopting a $2 \sigma$ upper limit for $\mathcal{T}$, we have followed the method used to measure existing limits on $\tau_{\mathrm{GP}}^{\text {eff }}$ (e.g. Fan et al. 2006a; Becker et al. 2015). Given that absorption in the $\operatorname{Ly} \alpha$ forest is saturated at $z \sim 6.5$, our choice is ultimately not very important.
}

transitions, as well as $\operatorname{Ly} \alpha$. In principle, observations of $\operatorname{Ly} \beta$ in the redshift range $6.61<z<7.08$ might provide a more sensitive measurement of the effective optical depth, if corrected for the coincident absorption by $\operatorname{Ly} \alpha$ at $5.47<z<5$.82. Although a statistical correction for foreground $\operatorname{Ly} \alpha$ is possible at lower redshifts, the recent results of Becker et al. (2015) show that this is not possible at $z \gtrsim 5.5$, as some lines of sight are completely absorbed in Ly $\alpha$.

The results for ULAS J1120+0641 lend support to the steadily-growing body of evidence that $\tau_{\mathrm{GP}}^{\mathrm{eff}}$ climbs rapidly at high redshift, and that therefore we are observing the tail-end of the epoch of reionization. However, it is also clear from Fig. 7 that we are unable to make especially useful measurements of $\tau_{\mathrm{GP}}^{\mathrm{eff}}$ for $z \gtrsim 6.5$. Absorption in the spectrum becomes saturated, and so no further information can be gained by measuring up to redshifts of seven since we can only determine lower limits which are similar in value to the measurements of $\tau_{\mathrm{GP}}^{\mathrm{eff}}$ from lower redshift sources. As expected, the most useful constraints on $\tau_{\mathrm{GP}}^{\mathrm{eff}}$ come from $z \sim 6$.

\section{Analysis and discussion}

\subsection{IGM opacity}

Fan et al. (2006a) used the spectra of $19 z>6$ quasars to measure $\tau_{\mathrm{GP}}^{\text {eff }}$ along different lines of sight. They found the best-fit power law to be:

$\tau_{\mathrm{GP}}^{\mathrm{eff}}(z)= \begin{cases}0.85\left(\frac{1+z}{5}\right)^{4.3} & z \leq 5.5 \\ 2.63\left(\frac{1+z}{6.5}\right)^{\xi} & z>5.5,\end{cases}$

empirically determining a limit of $\xi>10.9$. We now wish to measure a value of $\xi$ following a procedure similar to that used by Simpson et al. (2014), using our revised photometry. We have an additional constraint on $\xi$, namely the transmission spikes detected in the X-shooter spectrum.

The intrinsic quasar emission below Ly $\alpha$ is modelled using the power law chosen by Mortlock et al. (2011), $f_{\lambda}^{\text {intrinsic }} \propto \lambda^{-0.5}$, which is normalised to the observed spectrum at $1280 \AA$. Absorption in the IGM is modelled using Eq. (1). The wavelength of each pixel is converted to a $\operatorname{Ly} \alpha$ redshift, which is used to 


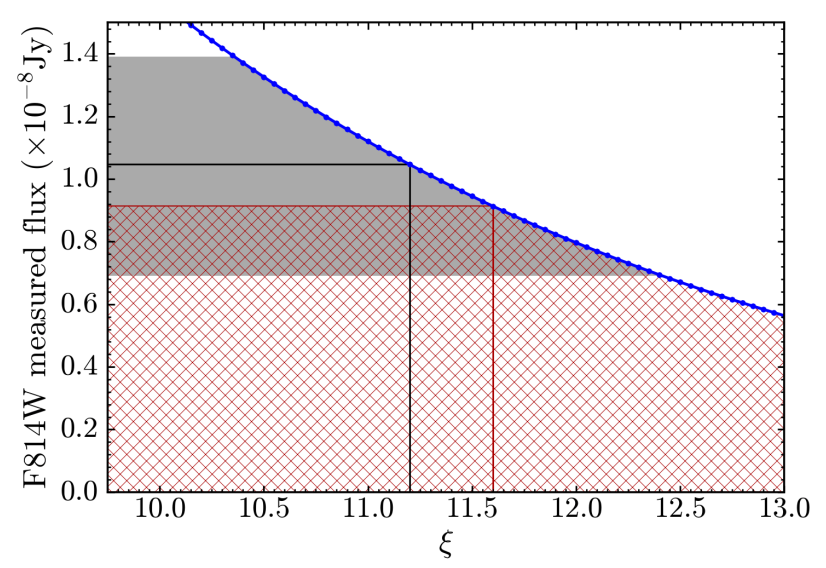

Fig. 8. Synthetic F $814 \mathrm{~W}$ photometry measured by determining $\tau_{\mathrm{GP}}^{\text {eff }}$ from different values of $\xi$ (Eq. (1)) is indicated by the blue points. The solid black line represents the $F 814 W$ flux in the HST ACS frame. The $1 \sigma$ range for the HST measurement is shaded grey. The red hatching indicates the hard lower limit on the measured $F 814 \mathrm{~W}$ flux imposed by the $\mathrm{X}$-shooter spectrum. The vertical red line indicates the resulting upper limit on $\xi$.

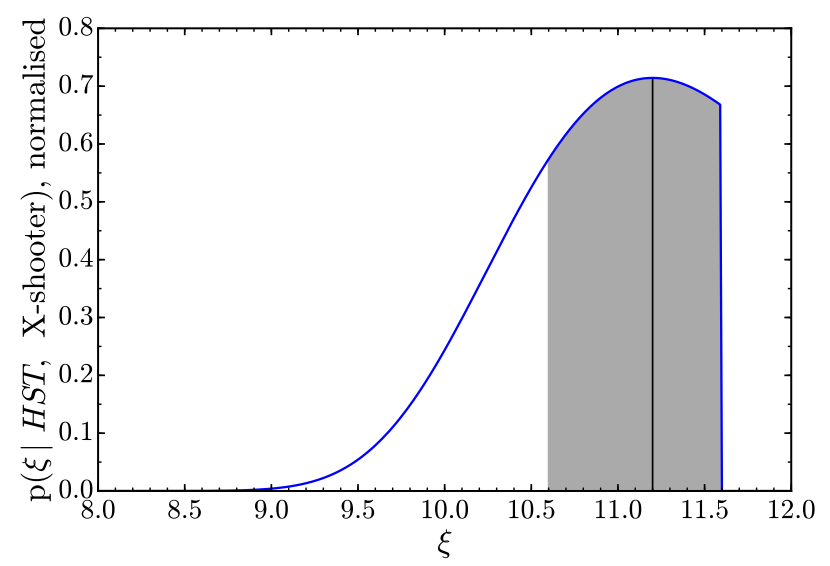

Fig. 9. Posterior distribution for $\xi$, constrained by photometry and spectroscopy. The $68 \%$ highest posterior density credible interval is shaded and the peak of the distribution is indicated at $\xi=11.2$. The cut-off at $\xi=11.6$ arises from determining a lower limit to the $i_{814}$ flux from the transmission spectrum derived in Sect. 2.1.

calculate $\tau_{\mathrm{GP}}^{\text {eff }}$ for a particular value of $\xi$. Measuring the absorbed spectrum with SYNPHOT produces a synthetic $i_{814}$ magnitude that we can compare directly to the $F 814 W$ result. We show the fluxes measured for a range of $\xi$ values in Fig. 8. Combining the synthetic photometry with spectroscopy, we determine a posterior distribution for $\xi$, shown in Fig. 9. We have assumed a uniform prior for $\xi>0$, and a Gaussian distribution for the $i_{814}$ measurement which is equivalent to $(1.047 \pm 0.356) \times 10^{-8} \mathrm{Jy}$. The sharp cut-off results from determining a lower limit to the $i_{814}$ flux from the transmission spectrum derived in Sect. 2.1, measured as $0.914 \times 10^{-8} \mathrm{Jy}(\mathrm{AB} 29.00, S / N \sim 30)$. Higher values of $\xi$ are excluded, as the resulting $i_{814}$ measurements are below this secure lower flux limit. The posterior peaks at $\xi=11.2$, for which the synthetic photometry yields the HST result (Fig. 9). The $68 \%$ highest posterior density credible interval is shaded, giving $\xi=11.2_{-0.6}^{+0.4}$. The resulting evolution of $\tau_{\mathrm{GP}}^{\mathrm{eff}}(z)$ is shown in Fig. 7.

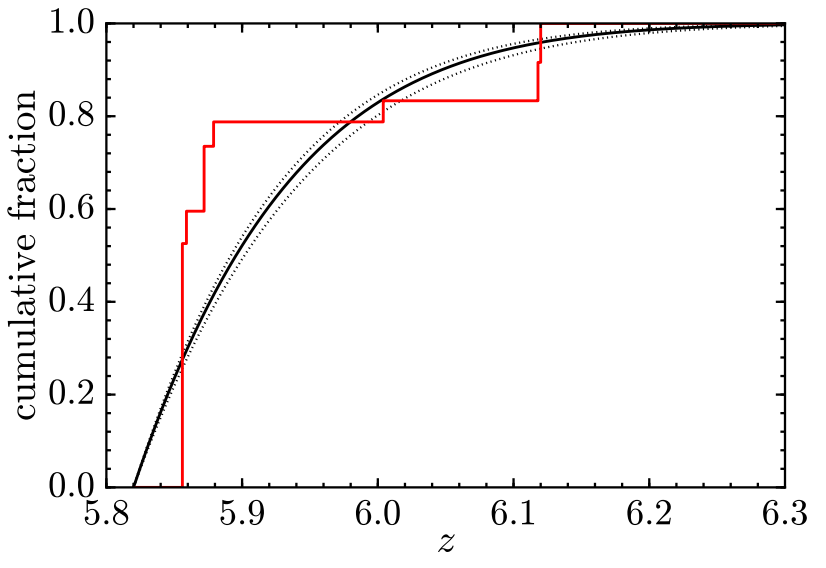

Fig. 10. Cumulative distribution of Ly $\alpha$ forest flux contained in the detected transmission spikes (red), compared with the expected distribution of flux transmission based on our model for optical depth with $\xi=11.2$ (black; Sect. 4.1). The dashed black lines indicate the uncertainty on our model for $\tau_{\mathrm{GP}}^{\mathrm{eff}}$.

\subsection{Flux distribution in the Ly $\alpha$ forest: the longest GP trough to date}

The detected transmission spikes account for $(87 \pm 34) \%$ of the quasar flux detected by HST, with the uncertainty dominated by the photometry measurement. Therefore all of the transmission in the spectrum may have been detected. Even if there are any additional lower-significance transmission features they are likely to be found in the same redshift range and to follow a similar distribution. The shape of the cumulative distribution of the transmitted flux therefore provides a noisy estimate of the shape of the cumulative transmission of the Ly $\alpha$ forest, and so it is interesting to compare this to the model.

In Fig. 10 we present the cumulative proportion of flux contained in transmission spikes with redshift i.e. the cumulative transmitted flux as a function of redshift, normalised to the total transmitted flux. The result from spectroscopy is compared to the $\tau_{\mathrm{GP}}^{\mathrm{eff}}$ model derived in Sect. 4.1, which we use to produce an average transmission spectrum. In both cases the distributions are normalised to the total transmitted flux as we wish to compare the relative shapes.

Broadly speaking, the distributions are in good agreement. In particular, our model for $\tau_{\mathrm{GP}}^{\mathrm{eff}}$ predicts effectively zero transmission for $z \gtrsim 6.2$, which is consistent with the transmission spectrum.

The distribution of transmission features is consistent with an extremely long GP trough extending to the quasar's nearzone, covering the redshift range $6.12 \leq z \leq 7.04$, equivalent to a comoving distance of $240 \mathrm{~h}^{-1} \mathrm{Mpc}$. This is more than twice the length of the next longest trough reported by Becker et al. (2015) towards the $z=5.98$ quasar ULAS J0148+0600, although that trough extends to significantly lower redshifts $(5.52 \leq z \leq 5.88)$.

\subsection{Constraints on the IGM neutral fraction}

Previous studies have used the evolution of the IGM Ly $\alpha$ opacity to constrain $x_{\mathrm{H} \text { I }}$ up to $z=6.3$ (Fan et al. 2006a; Becker et al. 2015). The large cross-section of $\operatorname{Ly} \alpha$ means that the forest will be almost completely absorbed by $z \sim 6$ even for $x_{\mathrm{H} \text { I }} \sim 10^{-4}$ (e.g. Fan et al. 2006a), and density evolution will naturally make the IGM even more opaque at $z>6$. Nevertheless, we wish to determine what we can learn about $x_{\mathrm{HI}}$ from the long trough towards ULAS J1120+0641. 
In order to constrain $x_{\mathrm{H}}$ we use artificial spectra generated from the Sherwood suite of hydrodynamical simulations, which are described in detail in Bolton et al. (2017). Briefly, they were run using a modified version of the parallel TreePM SPH code P-GADGET-3, which is an updated version of the publicly available GADGET-2 (Springel 2005). The models use a $\Lambda \mathrm{CDM}$ cosmology consistent with recent Planck results (Planck Collaboration XVI 2014). Here we use the reference 40-2048 model, which uses a $40 h^{-1} \mathrm{Mpc}$ box and $2 \times 2048^{3}$ particles, giving a gas particle mass of $9.97 \times 10^{4} M_{\odot}$. Reionization occurs at $z_{\mathrm{r}}=15$, whereafter the gas temperature and ionization fraction evolve assuming a spatially uniform Haardt \& Madau (2012) ionizing ultraviolet background (UVB). Simulation outputs were captured in redshift steps of $\Delta z=0.1$, and onedimensional Ly $\alpha$ forest skewers were generated from the outputs following standard methods (e.g., Theuns et al. 1998).

Our goal is to set limits on the neutral fraction required to produce the $240 h^{-1} \mathrm{Mpc}$ trough over $6.12 \leq z \leq 7.04$ seen towards ULAS J1120+0641, and we generate mock Ly $\alpha$ forest spectra with properties matched to the data using the following approach:

1. For each trial, six individual $40 h^{-1} \mathrm{Mpc}$ skewers are selected at random from the simulation, with the simulation redshifts chosen to most closely match the observed line-of-sight.

2. The Ly $\alpha$ optical depths $\left(\tau_{\alpha}\right)$ are scaled according to the ratio of our desired $x_{\mathrm{HI}}$ to the native $x_{\mathrm{HI}}$ in the simulation.

3. Transmitted fluxes are computed as $F=\mathrm{e}^{-\tau_{\alpha}}$.

4. The spectra are smoothed to the nominal instrumental resolution of $F W H M=34 \mathrm{~km} \mathrm{~s}^{-1}$ and binned using $10 \mathrm{~km} \mathrm{~s}^{-1}$ pixels, as in the real data.

5. Random Gaussian noise is added with dispersion given by the ULAS J1120+0641 error spectrum (normalized by a power law fit to the flux redward of the $\operatorname{Ly} \alpha$ emission line and extrapolated over the forest).

6. A matched-filter search for transmission peaks is then performed following the procedure described in Sect. 3.2. For efficiency, we use only the narrowest filter width $(\Sigma=$ $15 \mathrm{~km} \mathrm{~s}^{-1}$ ), although our results are not highly sensitive to this choice.

We parametrize the evolution in the neutral fraction as a power law of the form $x_{\mathrm{HI}}(z)=x_{0}[(1+z) / 7.1]^{\beta}$, where $x_{0}$ is the volumeweighted neutral fraction at $z=6.1$, i.e., near the start of the trough. For reference, the simulation has a native $x_{\mathrm{H}}$ evolution given by $x_{\mathrm{HI}}(z) \simeq\left(1.4 \times 10^{-4}\right)[(1+z) / 7.1]^{9.1}$ over the redshift range of interest. We generate $10^{4}$ mock spectra for each combination of $x_{0}$ and $\beta$. Our $95 \%$ confidence limits on these parameters are those for which no transmission peaks are detected with $S / N \geq 5$ in at least $5 \%$ of the trials. For $\beta=0$, we find $x_{0} \geq 1.4 \times 10^{-4}$. Increasing $\beta$ to 9 gives $x_{0} \geq 1.1 \times 10^{-4}$, which is consistent with using a Haardt \& Madau (2012) UVB. The limits on $x_{0}$ are thus largely insensitive to $\beta$, as expected if most of the constraining power comes from the low-redshift end of the trough.

In summary, we find that the Ly $\alpha$ trough towards ULAS J1120+0641, while remarkably long, provides only a weak lower limit to the volume-weighted neutral fraction of the IGM $\left(x_{\mathrm{HI}_{\mathrm{I}}} \gtrsim 10^{-4}\right)$, consistent with a highly ionized IGM. The weakness of this constraint is because the trough lies at very high redshift $(z \geq 6.1)$, where large Ly $\alpha$ opacities are more easily produced due to high IGM densities. We note that we have only considered an IGM model with a spatially uniform UVB and temperature-density relation, which implicitly assumes that the most transmissive regions of the IGM will be low-density voids. There is evidence that $x_{\mathrm{HI}}$ is significantly patchy on large scales at $z \gtrsim 5.5$ (Becker et al. 2015). Models that attribute this patchiness to a non-uniform background produced by star-forming galaxies tend to predict that the UVB intensity will be anti-correlated with large-scale density enhancements (Davies \& Furlanetto 2016; D'Aloisio et al. 2016). In these models, the highest transmission tends to come from high-density regions, and thus a lower $x_{\mathrm{HI}}$ would be required to produce significant Ly $\alpha$ transmission. Alternatively, the $x_{\mathrm{HI}}$ patchiness may be due to residual large-scale temperature fluctuations from an extended reionization process (D'Aloisio et al. 2015). In that model, high transmission tends to occur in hot ( $T \sim 20000-30000 \mathrm{~K})$, low-density regions that have only recently been reionized. Since these regions would be hotter than we have assumed and the neutral fraction scales as $T^{-0.7}$, Ly $\alpha$ transmission might appear even if the globally-averaged $x_{\mathrm{HI}}$ is somewhat larger than our constraint.

The constraints on $x_{\mathrm{HI}}$ from the Ly $\alpha$ trough towards ULAS J1120+0641 are considerably weaker than those determined from $\operatorname{Ly} \alpha$ damping wing absorption, first measured by Mortlock et al. (2011), who found $x_{\mathrm{HI}}>0.1$ at $z=7.1$. The most recent Ly $\alpha$ damping wing constraints on the volume-weighted neutral fraction come from an analysis of the FIRE infrared spectrometer spectrum of ULAS J1120+0641 (Simcoe et al. 2012) by Greig et al. (2016), who find $x_{\mathrm{HI}}(z=7.1)=0.4 \pm 0.2$. Greig \& Mesinger (2016) argue that the measured Ly $\alpha$ damping wing absorption towards ULAS J1120+0641 provides one of the strongest constraints on reionization that are currently available. As we begin to probe ever-higher neutral fractions, measurements of Ly $\alpha$ damping wing absorption will likely provide far more useful constraints than the Ly $\alpha$ forest. A further analysis of the damping wing towards ULAS J1120+0641 using the deeper X-shooter spectrum will be presented in Hewett et al. (in prep.).

\section{Summary}

We have analysed a deep X-shooter spectrum and HST F814W photometry of ULAS $\mathrm{J} 1120+0641$ to probe the Ly $\alpha$ forest to $z \simeq 7$ along the line of sight to the quasar. Our main results are as follows:

1. We revise $F 814 W$ photometry of ULAS J1120+0641 to be $i_{814}=28.85$, with $S / N=2.9$. For a faint source such as ULAS J1120+0641 which is measured against a high background, the Anderson \& Bedin (2010) CTE correction included in the ACS pipeline merely introduces noise to the photometry.

2. Significant transmission in the Lyman series forest is restricted to the redshift range $5.856<z<6.121$, where seven spikes were detected at $S / N>5$.

3. Photometry provides us with more useful constraints on effective optical depth, while spectroscopy can still be used to derive a lower limit on transmitted flux. Using data for ULAS J1120+0641 alone and assuming a fixed normalization at $z=5.5$, for redshifts $z>5.5$ we find $\tau_{\mathrm{GP}}^{\mathrm{eff}} \propto(1+z)^{\xi}$ where $\xi=11.2_{-0.6}^{+0.4}$.

4. We detect an extremely long dark gap extending from the final transmission spike to the quasar's near-zone, equivalent to a comoving distance of $240 h^{-1} \mathrm{Mpc}$. The GP trough nevertheless provides only a weak limit on the IGM neutral fraction $x_{\mathrm{HI}} \gtrsim 10^{-4}$ at $95 \%$ confidence. This is a generic limitation of observations of the Ly $\alpha$ forest at such high redshifts. 
R. Barnett et al.: Observations of the Lyman series forest towards the redshift 7.1 quasar ULAS J1120+0641

Acknowledgements. We are grateful to the referee, Michael Strauss, who provided constructive comments and improvements. We would like to thank the A.C.S. instrument team for their helpful guidance on the effects of CTE losses. We also thank Jamie Bolton for the use of the Sherwood simulations. This work was supported by Grants ST/K502042/1 and ST/N000838/1 from the Science and Technology Facilities Council. S.J.W. gratefully acknowledges the support of the Leverhulme Trust through the award of a Leverhulme Research Fellowship. G.D.B. is supported by the National Science Foundation under Grant AST 1615814. P.C.H. and R.G.M. acknowledge support from the STFC via a Consolidated Grant to the Institute of Astronomy, Cambridge. B.P.V. acknowledges funding through the ERC grant "Cosmic Dawn".

\section{References}

Anderson, J., \& Bedin, L. R. 2010, PASP, 122, 1035

Barnett, R., Warren, S. J., Banerji, M., et al. 2015, A\&A, 575, A31

Becker, R. H., Fan, X., White, R. L., et al. 2001, AJ, 122, 2850

Becker, G. D., Bolton, J. S., Madau, P., et al. 2015, MNRAS, 448, 19

Bertin, E., \& Arnouts, S. 1996, A\&AS, 117, 393

Bolton, A. S., Burles, S., Schlegel, D. J., Eisenstein, D. J., \& Brinkmann, J. 2004, AJ, 127, 1860

Bolton, J. S., Puchwein, E., Sijacki, D., et al. 2017, MNRAS, 464, 897

Bosman, S. E. I., Becker, G. D., Haehnelt, M. G., et al. 2017, MNRAS, in press

Chiaberge, M. 2012, Instrument Science Report ACS 2012-05

D'Aloisio, A., McQuinn, M., \& Trac, H. 2015, ApJ, 813, L38

D’Aloisio, A., McQuinn, M., Davies, F. B., \& Furlanetto, S. R. 2016, MNRAS submitted [arXiv: 1611.02711]

Davies, F. B., \& Furlanetto, S. R. 2016, MNRAS, 460, 1328

Djorgovski, S. G., Castro, S., Stern, D., \& Mahabal, A. A. 2001, ApJ, 560, L5

Fan, X., White, R. L., Davis, M., et al. 2000, AJ, 120, 1167
Fan, X., Narayanan, V. K., Lupton, R. H., et al. 2001, AJ, 122, 2833

Fan, X., Strauss, M. A., Becker, R. H., et al. 2006a, AJ, 132, 117

Fan, X., Carilli, C., \& Keating, B. 2006b, ARA\&A, 44, 415

Greig, B., \& Mesinger, A. 2016, MNRAS, 465, 4838

Greig, B., Mesinger, A., Haiman, Z., \& Simcoe, R. A. 2017, MNRAS, 466, 4239

Gunn, J. E., \& Peterson, B. A. 1965, ApJ, 142, 1633

Haardt, F., \& Madau, P. 2012, ApJ, 746, 125

Hewett, P., Irwin, M., Bunclark, P., et al. 1985, MNRAS, 213, 971

Horne, K. 1986, PASP, 98, 609

Kelson, D. D. 2003, PASP, 115, 688

Laidler, V., Boffi, F., Barlow, T., et al. 2008, Synphot Data User's Guide (Baltimore, STScI)

Lusso, E., Worseck, G., Hennawi, J. F., et al. 2015, MNRAS, 449, 4204

McGreer, I. D., Mesinger, A., \& Fan, X. 2011, MNRAS, 415, 3237

McGreer, I. D., Mesinger, A., \& D’Odorico, V. 2014, MNRAS, 447, 499

Mesinger, A. 2010, MNRAS, 407, 1328

Mortlock, D. J., Warren, S. J., Venemans, B. P., et al. 2011, Nature, 474, 616

Oke, J. B., \& Gunn, J. E. 1983, ApJ, 266, 713

Planck Collaboration XVI. 2014, A\&A, 571, A16

Planck Collaboration XLVI. 2016, A\&A, 596, A107

Planck Collaboration XLVII. 2016, A\&A, 596, A108

Schmidt, M. 1965, ApJ, 141, 1295

Simcoe, R. A., Sullivan, P. W., Cooksey, K. L., et al. 2012, Nature, 492, 79

Simpson, C., Mortlock, D., Warren, S., et al. 2014, MNRAS, 442, 3454

Songaila, A., \& Cowie, L. L. 2002, AJ, 123, 2183

Springel, V. 2005, MNRAS, 364, 1105

Theuns, T., Leonard, A., Efstathiou, G., Pearce, F. R., \& Thomas, P. A. 1998, MNRAS, 301, 478

Venemans, B. P., McMahon, R. G., Walter, F., et al. 2012, ApJ, 751, L25

Vernet, J., Dekker, H., D’Odorico, S., et al. 2011, A\&A, 536, A105

White, R. L., Becker, R. H., Fan, X., \& Strauss, M. A. 2003, AJ, 122, 34

White, R. L., Becker, R. H., Fan, X., \& Strauss, M. A. 2005, AJ, 129, 2102 